\title{
REMOVABLE SINGULARITIES OF SOLUTIONS OF NONLINEAR SINGULAR PARTIAL DIFFERENTIAL EQUATIONS
}

\author{
HIDETOSHI TAHARA \\ Department of Mathematics, Sophia University \\ Kioicho, Chiyoda-ku, 102 Tokyo, Japan \\ E-mail: h-tahara@hoffman.cc.sophia.ac.jp
}

1. Introduction. The study of singularities has been one of the main subjects of research in partial differential equations. In the case of linear equations the singularities are now pretty well understood; but in the nonlinear case there seems to be still very few studies.

In this paper I want to discuss the singularities of solutions of a class of nonlinear singular partial differential equations in the complex domain.

The class is only a model, but it helps one understand that the situation in the nonlinear case is more complicated than in the linear case.

2. Formulation. Consider the following type of nonlinear partial differential equation:

$$
\left(t \frac{\partial}{\partial t}\right)^{m} u=F\left(t, x,\left\{\left(t \frac{\partial}{\partial t}\right)^{j}\left(\frac{\partial}{\partial x}\right)^{\alpha} u\right\}_{\substack{j+|\alpha| \leq m \\ j<m}}\right)
$$

where

$$
\begin{gathered}
m \in\{1,2, \ldots\}, \quad t \in \mathbf{C}, \quad x=\left(x_{1}, \ldots, x_{n}\right) \in \mathbf{C}^{n}, \\
\alpha=\left(\alpha_{1}, \ldots, \alpha_{n}\right) \in\{0,1,2, \ldots\}^{n}, \quad|\alpha|=\alpha_{1}+\ldots+\alpha_{n}, \\
\left(\frac{\partial}{\partial x}\right)^{\alpha}=\left(\frac{\partial}{\partial x_{1}}\right)^{\alpha_{1}} \ldots\left(\frac{\partial}{\partial x_{n}}\right)^{\alpha_{n}},
\end{gathered}
$$

and for $F(t, x, Z)$ with $Z=\left\{Z_{j, \alpha}\right\}_{j+|\alpha| \leq m, j<m}$ we assume the following conditions:

$\left.\mathrm{A}_{1}\right) F(t, x, Z)$ is holomorphic in $(t, x, Z)$ near $(0,0,0)$;

$\left.\mathrm{A}_{2}\right) F(0, x, 0) \equiv 0$ near $x=0$;

$\left.\mathrm{A}_{3}\right)\left(\partial F / \partial Z_{j, \alpha}\right)(0, x, 0) \equiv 0$ near $x=0$, if $|\alpha|>0$.

1991 Mathematics Subject Classification: Primary 35A20; Secondary 35B40, 35C10.

The paper is in final form and no version of it will be published elsewhere. 
Rem ark 1 . When $F(t, x, Z)$ is linear in $Z$, equation $(\mathrm{E})$ is nothing but the Fuchsian type partial differential equation discussed in Tahara [5], [6]. When $m=1$, it is the Briot-Bouquet type partial differential equation discussed in Gérard-Tahara [1].

Under $\left.\left.A_{1}\right), A_{2}\right), A_{3}$ ) we define

$$
C(\varrho, x)=\varrho^{m}-\sum_{j<m} \frac{\partial F}{\partial Z_{j, 0}}(0, x, 0) \varrho^{j}
$$

and denote by $\varrho_{1}(x), \ldots, \varrho_{m}(x)$ the roots of $C(\varrho, x)=0$ in $\varrho$. We call these roots the characteristic exponents of $(\mathrm{E})$. We have:

Theorem 0 (Gérard-Tahara [2]). If $\varrho_{i}(0) \notin\{1,2, \ldots\}$ for any $i=1,2, \ldots, m$, then equation (E) has a unique solution $u(t, x)$ holomorphic in a neighborhood of $(0,0)$ and satisfying $u(0, x) \equiv 0$.

3. Problem. By Theorem 0 we see that in the generic case equation $(\mathrm{E})$ has one and only one holomorphic solution. This means that other solutions of (E) must be singular if they exist. Hence, if we want to characterize the equation by the property of solutions, we need to find the structure of all the singular solutions of (E). Thus the following problem naturally arises:

Problem. Determine all kinds of singularities which appear in the solutions of $(\mathrm{E})$.

If we could construct all the solutions of (E) explicitly, then the problem would be solved immediately.

But, in fact, it is very difficult and so it will be convenient to divide our problem into the following two parts:

(I) What kind of singularities are apparent?

(II) What kind of singularities can be admissible?

The problem (I) is the main theme of this paper. In Gérard-Tahara [2] some singular solutions are constructed explicitly, which contributes to the problem (II).

4. Result (1). We denote by:

- $\mathcal{R}(\mathbf{C} \backslash\{0\})$ the universal covering space of $\mathbf{C} \backslash\{0\}$;

- $S_{\theta}(\delta)=\{t \in \mathcal{R}(\mathbf{C} \backslash\{0\}) ; 0<|t|<\delta$ and $|\arg t|<\theta\}$;

- $D_{r}=\left\{x \in \mathbf{C}^{n} ;|x| \leq r\right\}$.

Put

Then we have:

$$
\varrho^{*}=\max _{1 \leq i \leq m} \operatorname{Re} \varrho_{i}(0)
$$

TheOREM 1. If $u(t, x)$ is a solution of $(\mathrm{E})$ holomorphic on $S_{\theta}(\delta) \times D_{r}$ for some $\theta>0$, $\delta>0, r>0$ and satisfying

$$
\max _{|x|<r}|u(t, x)|=O\left(|t|^{a}\right) \quad(\text { as } t \rightarrow 0)
$$

for some $a>\max \left\{\varrho^{*}, 0\right\}$, then $u(t, x)$ is holomorphic in a full neighborhood of $(0,0)$. 
Sketch of proof. Assume that there exists a solution $u(t, x)$ of (E) satisfying (4.1) for some $a>\max \left\{\varrho^{*}, 0\right\}$. Put $\ell=\min \{q \in \mathbf{Z} ; q \geq a\}$. Then it is easy to see that the condition

$$
F(t, x, 0)=O\left(t^{\ell}\right) \quad(\text { as } t \rightarrow 0)
$$

is satisfied and therefore equation (E) has a unique holomorphic solution $u_{0}(t, x)$ satisfying $u_{0}(t, x)=O\left(t^{\ell}\right)$ (as $\left.t \rightarrow 0\right)$. Since $a>0, a>\varrho^{*}$ and $\ell>\varrho^{*}$, by applying Proposition 3 in Gérard-Tahara [2] we can obtain $u(t, x) \equiv u_{0}(t, x)$ near $(0,0)$. This proves Theorem 1 .

Remark 2. (1) From Gérard-Tahara [2, Théorème 3] we know the following. Let $p$ be such that $\operatorname{Re} \varrho_{p}(0)=\varrho^{*}$. If

1) $\varrho_{p}(x)$ is holomorphic near $x=0$,

2) $\operatorname{Re} \varrho_{p}(0)=\varrho^{*}>0$,

3) $\varrho_{1}(0), \ldots, \varrho_{m}(0) \notin\{1,2, \ldots\}$,

then we can construct a singular solution of (E) which is holomorphic on $S_{\theta}(\delta) \times D_{r}$ for some $\theta>0, \delta>0, r>0$ and is of the form

$$
u(t, x)=\sum_{i \geq 1} u_{i}(x) t^{i}+\sum_{\substack{i+2 m j \geq k+2 m \\ j \geq 1}} \phi_{i, j, k}(x) t^{i+j \varrho_{p}(x)}(\log t)^{k}
$$

where $\phi_{0,1,0}(x)$ can be taken arbitrarily.

(2) The above singular solution $u(t, x)$ satisfies

$$
u(t, 0)=(\text { holomorphic part })+O\left(|t|^{\varrho^{*}}\right) \quad(\text { as } t \rightarrow 0) .
$$

This implies that in the case $\varrho^{*}>0$ the condition $a>\varrho^{*}$ is optimal in general.

Rem ark 3 . If equation (E) is linear, the condition $a>\max \left\{\varrho^{*}, 0\right\}$ can be replaced by $a>\varrho^{*}$ (see Tahara [5]). But in the nonlinear case it is impossible as is seen in the following example.

EXAMPLE 1. Let us consider

$$
t \frac{\partial u}{\partial t}+\varrho u=t u\left(\frac{\partial u}{\partial x}\right)^{k}
$$

where $(t, x) \in \mathbf{C}^{2}, \varrho>1 / k$ and $k \geq 1$ (integer). Then $\left(\mathrm{e}_{1}\right)$ has a solution

$$
u(t, x)=\left(\varrho-\frac{1}{k}\right)^{1 / k} \frac{x+c}{t^{1 / k}}, \quad c \in \mathbf{C} .
$$

In this case we have $\varrho^{*}=-\varrho<-1 / k$, and

$$
u(t, 0)=\text { const. } t^{-1 / k} \quad(\text { as } t \rightarrow 0) \text {. }
$$

5. Result (2). When $\varrho^{*}<0$, for the singularity of the form $(4.1)$ we have seen:

(1) (by Theorem 1) if $a>0$, the singularity is removable;

(2) (by Example 1) if $a<0$, the singularity is not removable in general.

The following theorem explains the situation near $a=0$. 
THEOREM 2. If $\varrho^{*}<0$ and if $u(t, x)$ is a solution of $(\mathrm{E})$ holomorphic on $S_{\theta}(\delta) \times D_{r}$ for some $\theta>0, \delta>0, r>0$ and satisfying

$$
\max _{|x|<r}|u(t, x)|=O\left(\frac{1}{(-\log |t|)^{\varepsilon}}\right) \quad(\text { as } t \rightarrow 0)
$$

for some $\varepsilon>0$, then $u(t, x)$ is holomorphic in a full neighborhood of $(0,0)$.

Sketch of proof. Let $u_{0}(t, x)$ be the unique holomorphic solution in Theorem 0 and let $u(t, x)$ be a solution of (E) satisfying (5.1) for some $\varepsilon>0$. Since $\varrho^{*}<0$, by the uniqueness theorem in Tahara [7] we can obtain $u(t, x) \equiv u_{0}(t, x)$ near $(0,0)$. This proves Theorem 2.

Re mark 4 . In the case $\varrho^{*}=0$, the singularity of the form (5.1) is not removable in general as is seen in the following example.

ExAmple 2. Let us consider

$$
t \frac{\partial u}{\partial t}=A u\left(\frac{\partial u}{\partial x}\right)^{k}
$$

where $(t, x) \in \mathbf{C}^{2}, A \neq 0$ and $k \geq 1$ (integer). Then $\left(\mathrm{e}_{2}\right)$ has a solution

$$
u(t, x)=\left(\frac{-1}{A k}\right)^{1 / k} \frac{x+c}{(\log t)^{1 / k}}, \quad c \in \mathbf{C} .
$$

Remark 5 . If $\operatorname{Re} \varrho_{i}(x) \leq 0$ (near $x=0$ ) for any $i=1,2, \ldots, m$, then we can prove that the singularity of the form (5.1) with $\varepsilon>m$ is removable. Compare this with the result in Example 2.

6. Supplementary remark. In this paper I have always assumed the conditions $A_{1}$ ), $A_{2}$ ) and $A_{3}$ ). Among them, the condition $A_{3}$ ) is essential to the theorem on removable singularities of type of Theorem 1 .

In this section, I will explain what happens if the condition $A_{3}$ ) is not satisfied.

Let us consider

$$
\left(t \frac{\partial}{\partial t}\right)^{m} u=F\left(t, x,\left\{\left(t \frac{\partial}{\partial t}\right)^{j}\left(\frac{\partial}{\partial x}\right)^{\alpha} u\right\}_{\substack{j+|\alpha| \leq m \\ j<m}}\right)
$$

and assume the condition $\left.\mathrm{A}_{1}\right)$. Put

$$
\begin{aligned}
\mathcal{J} & =\left\{(j, \alpha) ; \frac{\partial F}{\partial Z_{j, \alpha}}(0, x, 0) \not \equiv 0 \text { near } x=0\right\} ; \\
\mu & =\max \{|\alpha| ;(j, \alpha) \in \mathcal{J} \text { for some } j\} ; \\
\ell & =\max \{j ;(j, \alpha) \in \mathcal{J} \text { for some } \alpha \text { satisfying }|\alpha|=\mu\} .
\end{aligned}
$$

Remark 6. The condition $\mathrm{A}_{3}$ ) is equivalent to the condition $\mu=0$.

In the case $\mu \geq 1$, we have:

Proposition. Assume $\mathrm{A}_{1}$ ) and the following conditions:

$\left.c_{1}\right) F(t, x, 0) \equiv 0$ near $(t, x)=(0,0)$;

c. $\mu \geq 1$ 
$\left.c_{3}\right) \ell+\mu=m$

$\left.c_{4}\right)\left(\partial F / \partial Z_{\ell, \alpha}\right)(0,0,0) \neq 0$ for some $\alpha$ satisfying $|\alpha|=\mu$.

Then for any $\lambda>0$ sufficiently large we can find a singular solution $u_{\lambda}(t, x)$ of $\left(\mathrm{E}_{*}\right)$ which is holomorphic on $S_{\theta}(\delta) \times D_{r}$ for some $\theta>0, \delta>0, r>0$ and is of the form

$$
u_{\lambda}(t, x)=t^{\lambda}(\phi(x)+w(t, x)),
$$

where $\phi(x)$ and $w(t, x)$ are holomorphic functions on $S_{\theta}(\delta) \times D_{r}$ satisfying:

$$
\phi(0) \neq 0 ; \quad \max _{|x|<r}|w(t, x)|=o(1) \quad(\text { as } t \rightarrow 0) .
$$

Thus, if the condition $A_{3}$ ) is not satisfied, it seems difficult to get a theorem on removable singularities of type of Theorem 1 .

Remark 7 . When $F(t, x, Z)$ is algebraic in $Z$, similar singular solutions are constructed in Ishii [4]. We can prove the above proposition in the same way.

\section{References}

[1] R. Gérard and H. Tahara, Holomorphic and singular solutions of nonlinear singular first order partial differential equations, Publ. RIMS, Kyoto Univ. 26 (1990), 979-1000.

[2] -, -, Solutions holomorphes et singulières d'équations aux dérivées partielles singulières non linéaires, Publ. RIMS, Kyoto Univ. 29 (1993), 121-151.

[3] —, - Formal, holomorphic and singular solutions of nonlinear singular partial differential equations, to appear.

[4] T. Ishii, On propagation of regular singularities for nonlinear partial differential equations, J. Fac. Sci. Univ. Tokyo 37 (1990), 377-424.

[5] H. Tahara, Fuchsian type equations and Fuchsian hyperbolic equations, Japan. J. Math. 5 (1979), 245-347.

[6] -, Fundamental systems of analytic solutions of Fuchsian type partial differential equations, Funk. Ekvac. 24 (1981), 135-140.

[7] -, Uniqueness of the solution of nonlinear singular partial differential equations, J. Math. Soc. Japan 48 (1996), to appear. 